\title{
Some properties and applications of equicompact sets of operators
}

\author{
by \\ E. Serrano, C. Piñeiro and J. M. Delgado (Huelva)
}

\begin{abstract}
Let $X$ and $Y$ be Banach spaces. A subset $\mathrm{M}$ of $\mathcal{K}(X, Y)$ (the vector space of all compact operators from $X$ into $Y$ endowed with the operator norm) is said to be equicompact if every bounded sequence $\left(x_{n}\right)$ in $X$ has a subsequence $\left(x_{k(n)}\right)_{n}$ such that $\left(T x_{k(n)}\right)_{n}$ is uniformly convergent for $T \in \mathrm{M}$. We study the relationship between this concept and the notion of uniformly completely continuous set and give some applications. Among other results, we obtain a generalization of the classical Ascoli theorem and a compactness criterion in $\mathcal{M}_{\mathrm{c}}(\mathcal{F}, X)$, the Banach space of all (finitely additive) vector measures (with compact range) from a field $\mathcal{F}$ of sets into $X$ endowed with the semivariation norm.
\end{abstract}

1. Introduction. Throughout this paper $X$ and $Y$ will be Banach spaces. As usual, we will denote by $\mathcal{K}(X, Y)$ the Banach space of all compact operators from $X$ into $Y$ endowed with the operator norm. In [9] the authors introduced the notion of an equicompact set of operators. A set $\mathrm{M} \subset \mathcal{K}(X, Y)$ is said to be equicompact if every bounded sequence $\left(x_{n}\right)$ in $X$ has a subsequence $\left(x_{k(n)}\right)_{n}$ such that $\left(T x_{k(n)}\right)_{n}$ is uniformly convergent for $T \in \mathrm{M}$. They proved that the notions of equicompact set and collectively compact set are dual in the following sense: $\mathrm{M} \subset \mathcal{K}(X, Y)$ is equicompact (respectively, collectively compact) iff $\mathrm{M}^{*}=\left\{T^{*}: T \in \mathrm{M}\right\}$ is collectively compact (respectively, equicompact). We recall that $\mathrm{M}$ is called collectively compact if the set $\bigcup_{T \in \mathrm{M}} T\left(B_{X}\right)$ is relatively compact. Thus, the well known Palmer theorem [7] takes the following new form:

THEOREM A. If $\mathrm{M}$ is a subset of $\mathcal{K}(X, Y)$, then the following statements are equivalent:

(i) $\mathrm{M}$ is relatively compact.

(ii) $\mathrm{M}$ is equicompact and $\mathrm{M} x=\{T x: T \in \mathrm{M}\}$ is relatively compact for every $x \in X$.

2000 Mathematics Subject Classification: 47B07, 46G10.

Key words and phrases: compact operators, equicompact sets of operators, collectively compact set, vector measures, Ascoli's theorem. 
(iii) $\mathrm{M}$ is collectively compact and $\mathrm{M}^{*} y^{*}=\left\{T^{*} y^{*}: T \in \mathrm{M}\right\}$ is relatively compact for every $y^{*} \in Y^{*}$.

In particular, the authors of [9] have obtained the following characterization of compactness in a dual Banach space that we will use throughout this paper.

Corollary B. Let $X$ be a Banach space and $A \subset X^{*}$ a bounded set. Then $A$ is relatively compact iff every bounded sequence $\left(x_{n}\right)$ in $X$ has a subsequence $\left(x_{k(n)}\right)_{n}$ so that $\left(\left\langle x_{k(n)}, a\right\rangle\right)_{n}$ is uniformly convergent for $a \in A$.

In [9], the authors proved that a set $\mathrm{M} \subset \mathcal{K}(X, Y)$ is equicompact iff there exists a null sequence $\left(x_{n}^{*}\right)$ in $X^{*}$ such that $\|T x\| \leq \sup _{n}\left|\left\langle x, x_{n}^{*}\right\rangle\right|$ for all $x \in X$ and $T \in \mathrm{M}$. They also proved that equicompact sets are uniformly completely continuous, that is, $\left\|T x_{n}\right\| \rightarrow 0$ uniformly for $T \in \mathrm{M}$ whenever $\left(x_{n}\right)$ is a weakly null sequence in $X$. Actually, if the Banach space $X$ does not contain a copy of $\ell_{1}$, equicompact sets and uniformly completely continuous sets are the same (see Proposition 2.2 below).

In this paper we deepen the study of the relationships between equicompact and uniformly completely continuous sets. Moreover, we obtain a generalization of the classical Ascoli theorem and a characterization of compactness in $\mathcal{M}_{\mathrm{c}}(\mathcal{F}, X)$, the Banach space of all (finitely additive) vector measures from $\mathcal{F}$ into $X$ with compact range, $\mathcal{F}$ being a field of subsets of a set $\Omega$.

We use the classical notation in Banach space theory. If $X$ is a Banach space, $X^{*}$ denotes its dual space, $B_{X}$ its closed unit ball and $S_{X}$ its unit sphere. For a subset $A$ of $X, \overline{\mathrm{co}}(A)$ is the closed convex hull of $A$. As usual, $\ell_{1}(I, X)$ (respectively $\ell_{\infty}(I, X)$ ) stands for the Banach space of all functions $\widehat{x}: I \rightarrow X$ satisfying $\sum_{i \in I}\|\widehat{x}(i)\|<\infty$ (respectively $\left.\sup \{\|\widehat{x}(i)\|: i \in I\}<\infty\right)$ endowed with its natural norm. We will use the following version of the well known Vala compactness criterion in $\ell_{\infty}^{c}(I, X)$ (the Banach space of all functions $\widehat{x}: I \rightarrow X$ with relatively compact range endowed with the supremum norm).

Theorem 1.1 (K. Vala [10, Theorem 1]). Let $\mathrm{M} \subset \ell_{\infty}^{\mathrm{c}}(I, X)$ be bounded. The following statements are equivalent:

(i) $\mathrm{M}$ is relatively compact.

(ii) M has the following properties:

(a) For every $\varepsilon>0$ there exists a finite partition $\left\{D_{1}, \ldots, D_{p}\right\}$ of $I$ such that

$$
1 \leq k \leq p, i, j \in D_{k} \Rightarrow\|\widehat{x}(i)-\widehat{x}(j)\|<\varepsilon \text { for all } \widehat{x} \in \mathrm{M} .
$$

(b) $\mathrm{M}(i)=\{\widehat{x}(i): \widehat{x} \in \mathrm{M}\}$ is relatively compact for all $i \in I$.

Our notation from vector measure theory follows [3]. We only consider vector measures defined on fields of sets. If $\mathcal{F}$ is a field of subsets of a set $\Omega$, 
$X$ is a Banach space and $m: \mathcal{F} \rightarrow X$ is such a measure, we denote by $\|m\|(A)$ the semivariation of $A \in \mathcal{F}$ :

$$
\|m\|(A)=\sup \left\{\left|x^{*} \circ m\right|(A): x^{*} \in B_{X^{*}}\right\} .
$$

The range of $m$ is denoted by $\operatorname{rg}(m)$, that is, $\operatorname{rg}(m)=\{m(A): A \in \mathcal{F}\}$. Finally, we denote by $\mathcal{B}(\mathcal{F})$ the Banach space of all scalar-valued functions on $\Omega$ that are uniform limits of simple functions modeled on $\mathcal{F}$.

\section{Relationships between equicompact and uniformly comple- tely continuous sets}

TheOREM 2.1. Let $\mathrm{M}$ be a bounded subset of $\mathcal{K}(X, Y)$. The following statements are equivalent:

(i) $\mathrm{M}$ is equicompact.

(ii) M has the following properties:

(a) $\mathrm{M}$ is uniformly completely continuous.

(b) For every seminormalized sequence $\left(x_{n}\right)$ in $X$ equivalent to the $\ell_{1}$ unit vector basis and every $\varepsilon>0$, there exists a finite partition $\left\{D_{1}, \ldots, D_{p}\right\}$ of $\mathbb{N}$ such that $\left\|T x_{n}-T x_{m}\right\|<\varepsilon$ for all $T \in \mathrm{M}$ whenever $m, n \in D_{i}$ and $i=1, \ldots, p$.

Proof. (i) $\Rightarrow$ (ii). We only have to prove (b). Let $\phi: \ell_{1} \rightarrow \overline{\operatorname{span}}\left\{x_{n}: n \in \mathbb{N}\right\}$ be an isomorphism with $\phi\left(e_{n}\right)=x_{n}$ for all $n \in \mathbb{N}$. Obviously, the set $\mathrm{M} \circ \phi=$ $\{T \circ \phi: T \in \mathrm{M}\}$ is equicompact and, therefore, $\phi^{*} \circ \mathrm{M}^{*}$ is collectively compact. That is, the set

$$
\bigcup_{T \in \mathrm{M}} \phi^{*}\left(T^{*}\left(B_{Y^{*}}\right)=\left\{\left(\left\langle x_{n}, T^{*} y^{*}\right\rangle\right): y^{*} \in B_{Y^{*}}, T \in \mathrm{M}\right\}\right.
$$

is relatively compact in $\ell_{\infty}$. According to Theorem 1.1 , given $\varepsilon>0$, there exists a finite partition $\left\{D_{1}, \ldots, D_{p}\right\}$ of $\mathbb{N}$ so that

$$
n, m \in D_{i} \Rightarrow\left|\left\langle x_{n}-x_{m}, T^{*} y^{*}\right\rangle\right|<\varepsilon \text { for all } y^{*} \in B_{Y^{*}} \text { and } T \in \mathrm{M},
$$

for $i=1, \ldots, p$. This yields $\left\|T x_{n}-T x_{m}\right\|<\varepsilon$ for all $T \in \mathrm{M}$ and $i=1, \ldots, p$.

(ii) $\Rightarrow$ (i). Let $\left(x_{n}\right)$ be a bounded sequence in $X$. By Rosenthal's $\ell_{1}$ theorem, $\left(x_{n}\right)$ has a subsequence which is either weakly Cauchy or equivalent to the unit basis of $\ell_{1}$ (for simplicity, we will go on denoting it by $\left(x_{n}\right)$ ). In the first case, $\left(T x_{n}\right)$ is uniformly convergent for $T \in \mathrm{M}$ because $\mathrm{M}$ is uniformly completely continuous. In the second case, by hypothesis, there exists a partition $\left\{D_{1}, \ldots, D_{p}\right\}$ of $\mathbb{N}$ so that

$$
n, m \in D_{i} \Rightarrow\left\|T x_{n}-T x_{m}\right\|<1 \text { for all } T \in \mathrm{M}
$$

and $i=1, \ldots, p$. Some of the $D_{i}$ 's must be infinite, so we can choose $i \leq p$ such that $D_{i}$ is infinite. If $k_{1}: \mathbb{N} \rightarrow D_{i}$ is an increasing bijection, then $\left(x_{k_{1}(n)}\right)$ is a sequence equivalent to the unit basis of $\ell_{1}$; so repeating this process 
inductively, we can determine a sequence of subsequences $\left(k_{p}(n)\right)_{n}$ such that $\left(k_{p+1}(n)\right)_{n}$ is a subsequence of $\left(k_{p}(n)\right)_{n}$ and

$$
\left\|T x_{k_{p}(n)}-T x_{k_{p}(m)}\right\|<1 / p \quad \text { for all } n, m \in \mathbb{N} \text { and } T \in \mathrm{M}
$$

for all $p \in \mathbb{N}$. Now it is easy to deduce that $\left(T x_{k_{p}(p)}\right)_{p}$ is uniformly convergent for $T \in \mathrm{M}$.

The next proposition proves that all uniformly completely continuous sets are equicompact iff $X$ does not contain a copy of $\ell_{1}$. We denote by $\mathcal{V}(X, Y)$ the vector space of all completely continuous operators from $X$ into $Y$ endowed with the operator norm.

Proposition 2.2. Let $X$ be a Banach space. The following statements are equivalent:

(i) For every Banach space $Y$ and every $\mathrm{M} \subset \mathcal{V}(X, Y), \mathrm{M}$ is equicompact whenever $\mathrm{M}$ is uniformly completely continuous.

(ii) There exists a Banach space $Y$ such that every uniformly completely continuous set $\mathrm{M} \subset \mathcal{K}(X, Y)$ is equicompact.

(iii) $X$ does not contain copy of $\ell_{1}$.

Proof. If $X \nLeftarrow \ell_{1}$, then $\mathcal{V}(X, Y)=\mathcal{K}(X, Y)$ for all Banach spaces $Y$ and (iii) $\Rightarrow$ (i) can be deduced using Theorem 2.1; so we only have to prove (ii) $\Rightarrow$ (iii). Assuming (ii), to prove that $X$ does not contain a copy of $\ell_{1}$, we show that every uniformly completely continuous subset $A$ of $X^{*}$ is relatively compact [5, Th. 2]. Take $y_{0} \in S_{Y}$ and put $\mathrm{M}=A \otimes y_{0}$. It is obvious that $\mathrm{M}$ is a uniformly completely continuous subset of $\mathcal{K}(X, Y)$. So, by hypothesis, $\mathrm{M}$ is equicompact, which yields the equicompactness of $A$ as a subset of $\mathcal{K}(X, \mathbb{R})$. Finally, a call to Corollary B tells us that $A$ is relatively compact.

Recall that an operator $T: X \rightarrow Y$ is said to be conditionally weakly compact if every bounded sequence $\left(x_{n}\right)$ in $X$ admits a subsequence $\left(x_{k(n)}\right)_{n}$ so that $\left(T x_{k(n)}\right)_{n}$ is weakly Cauchy. We denote by $\mathcal{C} W(X, Y)$ the vector space of all conditionally weakly compact operators from $X$ into $Y$.

Proposition 2.3. For an operator $Q \in \mathcal{L}(X, Z)$, the following statements are equivalent:

(i) $Q \in \mathrm{e} W(X, Z)$.

(ii) If $A \subset Z^{*}$ is uniformly completely continuous, then $Q^{*}(A)$ is relatively compact.

(iii) For every Banach space $Y$ and every $\mathrm{N} \subset \mathcal{V}(Z, Y), \mathrm{N} \circ Q$ is equicompact whenever $\mathrm{N}$ is uniformly completely continuous.

Proof. (i) $\Rightarrow$ (ii). Let $A \subset Z^{*}$ be uniformly completely continuous and $Q \in \mathcal{C} W(X, Z)$. Given a bounded sequence $\left(x_{n}\right)$ in $X$, there exists a subsequence $\left(x_{k(n)}\right)_{n}$ such that $\left(Q x_{k(n)}\right)_{n}$ is weakly Cauchy. Then $\left(\left\langle Q x_{k(n)}, a\right\rangle\right)_{n}=$ 
$\left(\left\langle x_{k(n)}, Q^{*} a\right\rangle\right)_{n}$ is uniformly convergent for $a \in A$. Now, Corollary B concludes the proof.

(ii) $\Rightarrow$ (iii). Let $Y$ be a Banach space and $\mathrm{N} \subset \mathcal{V}(Z, Y)$ uniformly completely continuous. We prove that $Q^{*} \circ \mathrm{N}^{*}$ is collectively compact. For this, take a sequence $\left(\left(Q^{*} \circ S_{n}^{*}\right) y_{n}^{*}\right)_{n}$ in $\bigcup_{S \in \mathrm{N}} Q^{*} \circ S^{*}\left(B_{Y^{*}}\right)$ and put $A=\left\{S_{n}^{*} y_{n}^{*}\right.$ : $n \in \mathbb{N}\}$. The set $A$ is uniformly completely continuous. In fact, if $\left(z_{n}\right)$ is a weakly null sequence in $Z$, we have

$$
\left|\left\langle z_{n}, S_{m}^{*} y_{m}^{*}\right\rangle\right|=\left|\left\langle S_{m} z_{n}, y_{m}^{*}\right\rangle\right| \leq\left\|S_{m} z_{n}\right\| .
$$

Then, by hypothesis, the set $Q^{*}(A)$ is relatively compact and, therefore, $\left(\left(Q^{*} \circ S_{n}^{*}\right) y_{n}^{*}\right)_{n}$ has a convergent subsequence.

(iii) $\Rightarrow\left(\right.$ i). By hypothesis, $S\left(Q\left(B_{X}\right)\right)$ is relatively compact for all Banach space $Y$ and all $S \in \mathcal{V}(Z, Y)$. According to [8, p. 377], the set $Q\left(B_{X}\right)$ is conditionally weakly compact.

The next theorem shows that every equicompact set $\mathrm{M}$ admits a representation of the form $\mathrm{M}=\mathrm{N} \circ Q$, where $\mathrm{N}$ is uniformly completely continuous and $Q$ is conditionally weakly compact.

TheOREM 2.4. Let $\mathrm{M}$ be a subset of $\mathcal{L}(X, Y)$. The following statements are equivalent:

(i) $\mathrm{M}$ is equicompact.

(ii) There exist a closed subspace $Z$ of $c_{0}, Q \in \mathcal{K}(X, Z)$ and $\mathrm{N} \subset$ $\mathcal{K}(Z, Y)$ such that $\mathrm{N}$ is equicompact and $\mathrm{M}=\mathrm{N} \circ Q$.

(iii) There exist a Banach space $Z, Q \in \mathcal{C} W(X, Z)$ and $\mathrm{N} \subset \mathcal{V}(Z, Y)$ such that $\mathrm{N}$ is uniformly completely continuous and $\mathrm{M}=\mathrm{N} \circ Q$.

Proof. Only (i) $\Rightarrow$ (ii) needs to be proved. According to [9, Prop. 2.2], the equicompactness of $\mathrm{M}$ implies that there exists a null sequence $\left(x_{n}^{*}\right)$ in $X^{*}$ so that

$$
\|T x\| \leq \sup _{n}\left|\left\langle x, x_{n}^{*}\right\rangle\right| \quad \text { for all } x \in X \text { and } T \in \mathrm{M} .
$$

For each $n \in \mathbb{N}$, we define $\lambda_{n}=\sqrt{\left\|x_{n}^{*}\right\|}$ and $b_{n}^{*}=\lambda_{n}^{-1} x_{n}^{*}$ (we can assume that $\lambda_{n} \neq 0$ for all $n \in \mathbb{N}$ ). Obviously, $\lambda_{n} \rightarrow 0$ and $\left\|b_{n}^{*}\right\| \rightarrow 0$. Now, in a similar way to the proof of $[6$, Th. 17.1.4], we find a closed subspace $Z$ of $c_{0}$ and operators $Q \in \mathcal{K}(X, Z)$ and $S_{T} \in \mathcal{K}(Z, Y)$ satisfying $T=S_{T} \circ Q$, for all $T \in \mathrm{M}\left(Q: x \in X \mapsto\left(\left\langle x, b_{n}^{*}\right\rangle\right) \in c_{0}, Z=\overline{\{Q x: x \in X\}}\right.$ and $\left.S_{T}\left(\left\langle x, b_{n}^{*}\right\rangle\right)=T x\right)$.

Put $\mathrm{N}=\left\{S_{T}: T \in \mathrm{M}\right\}$. Since $Z \hookrightarrow c_{0}$, we have $Z^{*} \approx \ell_{1} / Z^{\perp}$. If $\left(e_{n}\right)$ denotes the unit vector basis of $\ell_{1}$, it is clear that

$$
\left\langle\left(\left\langle x, b_{n}^{*}\right\rangle\right), \lambda_{m}\left[e_{m}\right]\right\rangle=\left\langle x, \lambda_{m} b_{m}^{*}\right\rangle=\left\langle x, x_{m}^{*}\right\rangle \quad \text { and } \quad\left\|\lambda_{n}\left[e_{n}\right]\right\| \rightarrow 0 .
$$


Then, for all $T \in \mathrm{M}$, we have

$$
\left\|S_{T}\left(\left\langle x, b_{n}^{*}\right\rangle\right)\right\|=\|T x\| \leq \sup _{m}\left|\left\langle x, x_{m}^{*}\right\rangle\right|=\sup _{m}\left|\left\langle\left(\left\langle x, b_{n}^{*}\right\rangle\right), \lambda_{m}\left[e_{m}\right]\right\rangle\right|,
$$

that is, $\mathrm{N}$ is equicompact. Finally, notice that $\mathrm{M}=\mathrm{N} \circ Q$.

3. A generalization of the classical Ascoli theorem. In this section we generalize the notion of equicompact set to a wider class of functions. Let $J$ be an arbitrary set and $Z$ a complete metric space. If $\mathrm{M}$ is a set of functions from $J$ into $Z$ with relatively compact range, we say that $M$ is equicompact if every sequence $\left(j_{n}\right)$ in $J$ has a subsequence $\left(j_{k(n)}\right)_{n}$ such that $\left(f\left(j_{k(n)}\right)\right)_{n}$ is uniformly convergent for $f \in \mathrm{M}$.

If $\mathcal{M} \subset \mathcal{K}(X, Y)$, where $X$ and $Y$ are Banach spaces, then $\mathcal{M}$ is equicompact (in the original sense) iff $\mathrm{M}=\left\{\left.T\right|_{B_{X}}: T \in \mathcal{M}\right\}$ is equicompact. Throughout this section $X$ will be a Banach space and $I$ an infinite index set. The mapping $\psi: \widehat{x} \in \ell_{\infty}^{\mathrm{c}}(I, X) \mapsto \psi(\widehat{x})=T_{\widehat{x}} \in \mathcal{K}\left(\ell_{1}(I), X\right)$ defined by $T_{\widehat{x}}\left(\xi_{i}\right)_{i \in I}=\sum_{i \in I} \xi_{i} \widehat{x}(i)$ is an isometric isomorphism. Using a similar argument to the proof of Theorem 2.1, it is easy to prove the next lemma:

Lemma 3.1. Let $\mathcal{M}$ be a bounded subset of $\mathcal{K}\left(\ell_{1}(I), X\right)$. Then $\mathcal{M}$ is equicompact iff for every $\varepsilon>0$ there exists a finite partition $\left\{D_{1}, \ldots, D_{p}\right\}$ of I such that

$$
1 \leq k \leq p, i, j \in D_{k} \Rightarrow\left\|T e_{i}-T e_{j}\right\|<\varepsilon \text { for all } T \in \mathcal{M} .
$$

REMARK 3.2. If $\psi(\mathrm{M}) \subset \mathcal{K}\left(\ell_{1}(I), X\right)$ is equicompact, then $\mathrm{M}$ is equicompact and bounded, but, in general, an equicompact set $\mathrm{M}$ in $\ell_{\infty}^{\mathrm{c}}(I, X)$ is not necessarily bounded. To see this, take an equicompact and bounded sequence $\left(\widehat{x}_{k}\right)$ in $\ell_{\infty}^{\mathrm{c}}(I, X)$ and choose $x_{0} \in S_{X}$. Now, for each $k \in \mathbb{N}$, denote by $\widehat{z}_{k} \in \ell_{\infty}^{\mathrm{c}}(I, X)$ the function defined by $\widehat{z}_{k}(i)=\widehat{x}_{k}(i)+k x_{0}$ for all $i \in I$. It is easy to prove that $\left(\widehat{z}_{k}\right)$ is an equicompact sequence but, nevertheless, it is not bounded.

Proposition 3.3. Let $\mathrm{M}$ be a bounded subset of $\ell_{\infty}^{\mathrm{c}}(I, X)$. The following statements are equivalent:

(i) $\mathrm{M}$ is equicompact.

(ii) $\psi(\mathrm{M})$ is equicompact.

(iii) There exists a null sequence $\left(\widehat{\beta}_{n}\right)$ in $\ell_{\infty}(I)$ so that

$$
\|\widehat{x}(i)-\widehat{x}(j)\| \leq \sup _{n}\left|\widehat{\beta}_{n}(i)-\widehat{\beta}_{n}(j)\right| \quad \text { for all } i, j \in I \text { and } \widehat{x} \in \mathrm{M} \text {. }
$$

Proof. (i) $\Rightarrow$ (ii). Consider the operator $U: \ell_{1}(I) \rightarrow \ell_{\infty}(\mathrm{M}, X)$ defined by $U\left(e_{i}\right)=(\widehat{x}(i))_{\widehat{x} \in \mathrm{M}}$ for all $i \in I\left(\left(e_{i}\right)_{i \in I}\right.$ is the canonical basis of $\left.\ell_{1}(I)\right)$. By (i), $U$ is compact and, therefore, the set $\psi(\mathrm{M})$ is equicompact [9, Prop. 2.2]. 
(ii) $\Rightarrow$ (iii). According to $[9$, Prop. 2.2$]$, there exists a null sequence $\left(\widehat{\beta}_{n}\right)$ in $\ell_{\infty}(I)$ satisfying $\left\|T_{\widehat{x}}(\xi)\right\| \leq \sup _{n}\left|\left\langle\xi, \widehat{\beta}_{n}\right\rangle\right|$ for all $\xi \in \ell_{1}(I)$ and $\widehat{x} \in \mathrm{M}$. In particular, for $i, j \in I$ we have $\|\widehat{x}(i)-\widehat{x}(j)\| \leq \sup _{n}\left|\widehat{\beta}_{n}(i)-\widehat{\beta}_{n}(j)\right|$ for all $\widehat{x} \in \mathrm{M}$.

(iii) $\Rightarrow(\mathrm{i})$. Given a sequence $\left(i_{n}\right)$ in $I$, there exists a subsequence $\left(i_{k(n)}\right)_{n}$ such that $\left(\left\langle e_{i_{k(n)}}, \widehat{\beta}_{m}\right\rangle\right)_{n}$ is uniformly convergent for $m \in \mathbb{N}$ because of the compactness of $\left\{\widehat{\beta}_{m}: m \in \mathbb{N}\right\}$ (Corollary B). Now, from the uniform convergence of $\left(\widehat{\beta}_{m}\left(i_{k(n)}\right)\right)_{n}$ for $m \in \mathbb{N}$ and (iii), it is easy to obtain (i).

REMARK 3.4. According to Lemma 3.1 and Proposition 3.3, a bounded subset $\mathrm{M}$ of $\ell_{\infty}^{\mathrm{c}}(I, X)$ is equicompact iff it satisfies condition (ii)(a) in Vala's theorem (Th. 1.1). As usual, if $\Omega$ is a compact topological space, $\mathrm{C}(\Omega, X)$ is the Banach space of all continuous functions $\phi: \Omega \rightarrow X$ endowed with the supremum norm. Obviously, $\mathrm{C}(\Omega, X)$ is a subspace of $\ell_{\infty}^{\mathrm{c}}(\Omega, X)$.

Now we are ready to show the main result of this section: a generalization of the classical Ascoli theorem [4, Th. 7.5.7].

THEOREM 3.5. Let $\mathrm{M}$ be a subset of $\mathrm{e}(\Omega, X)$, $\Omega$ being an arbitrary compact topological space. The following statements are equivalent:

(i) $\mathrm{M}$ is relatively compact.

(ii) M has the following properties:

(a) $\mathrm{M}$ is equicompact.

(b) $\mathrm{M}(\omega)=\{\phi(\omega): \phi \in \mathrm{M}\}$ is relatively compact for all $\omega \in \Omega$.

Proof. (i) $\Rightarrow$ (ii). follows directly from Theorem 1.1, Proposition 3.3 and Lemma 3.1. According to Remark 3.4, to prove (ii) $\Rightarrow$ (i) we only need to show that $\mathrm{M}$ is bounded. To see this, consider the function $F: \omega \in \Omega \mapsto$ $F(\omega)=(\phi(\omega))_{\phi \in \mathrm{M}} \in \ell_{\infty}(\mathrm{M}, X)$. Then $F$ is well defined and has compact range since $\mathrm{M}$ is equicompact.

The next proposition lists some elementary properties of equicompact sets of functions that allow us to consider the above theorem as a generalization of the classical Ascoli-Arzelà theorem.

Proposition 3.6. Let $\Omega$ be an arbitrary compact topological space and $\mathrm{M}$ a bounded subset of $\mathrm{C}(\Omega, X)$.

(1) If $\mathrm{M}$ is equicompact, then it is sequentially equicontinuous.

(2) If, in addition, $\Omega$ is metrizable, then $\mathrm{M}$ is equicompact iff it is equicontinuous.

Proof. (1) Suppose $\left(\omega_{n}\right)$ is a sequence in $\Omega$ with limit $\omega_{0} \in \Omega$. By continuity, for each $\phi \in \mathrm{M}$, we have $\phi\left(\omega_{n}\right) \rightarrow \phi\left(\omega_{0}\right)$. As M is equicompact, by contradiction, it is easy to prove that $\phi\left(\omega_{n}\right) \rightarrow \phi\left(\omega_{0}\right)$ uniformly in $\phi \in \mathrm{M}$. 
(2) In case $\Omega$ is metrizable, equicontinuity and sequential equicontinuity are the same. So, we only have to prove the sufficiency. Assume M is equicontinuous. Given a sequence $\left(\omega_{n}\right)$ in $\Omega$, as $\Omega$ is metrizable and compact, there is a convergent subsequence $\left(\omega_{k(n)}\right)_{n}$. Suppose that $\omega_{k(n)} \rightarrow \omega_{0} \in \Omega$. Since $\mathrm{M}$ is equicontinuous it follows that $\phi\left(\omega_{k(n)}\right) \rightarrow \phi\left(\omega_{0}\right)$ as $n \rightarrow \infty$ uniformly in $\phi \in \mathrm{M}$.

4. Compactness in $\mathcal{M}_{\mathrm{c}}(\mathcal{F}, X)$. As in Section 3, we say that a set $\mathrm{M} \subset$ $\mathcal{M}_{\mathrm{c}}(\mathcal{F}, X)$ is equicompact if every sequence $\left(A_{n}\right)$ in $\mathcal{F}$ has a subsequence $\left(A_{k(n)}\right)_{n}$ such that $\left(m\left(A_{k(n)}\right)\right)_{n}$ is uniformly convergent for $m \in \mathrm{M}$.

By [3, I.5.3], all vector measures in $\mathcal{M}_{\mathrm{c}}(\mathcal{F}, X)$ are strongly additive. To start, we prove that equicompact sets of vector measures are uniformly strongly additive. We recall that a set $\mathrm{M}$ of strongly additive vector measures is called uniformly strongly additive if, for every sequence $\left(A_{n}\right)$ of pairwise disjoint members of $\mathcal{F}, \lim _{n \rightarrow \infty}\left\|\sum_{k=n}^{\infty} m\left(A_{k}\right)\right\|=0$ uniformly in $m \in \mathrm{M}$.

Proposition 4.1. If $\mathrm{M}$ is an equicompact set of vector measures, then it is uniformly strongly additive.

Proof. According to [3, Proposition I.1.17], we have to prove that $\lim _{n \rightarrow \infty}\left\|m\left(A_{n}\right)\right\|=0$ uniformly in $m \in \mathrm{M}$ whenever $\left(A_{n}\right)$ is a sequence of pairwise disjoint members of $\mathcal{F}$. Arguing by contradiction, suppose there exist $\varepsilon>0$, a sequence $\left(m_{n}\right)$ in $\mathrm{M}$ and a subsequence $\left(A_{k(n)}\right)_{n}$ so that

$$
\left\|m_{n}\left(A_{k(n)}\right)\right\|>\varepsilon \quad \text { for all } n \in \mathbb{N} \text {. }
$$

By hypothesis, $\left(A_{k(n)}\right)_{n}$ has a subsequence $\left(A_{h(n)}\right)_{n}$ such that $\lim _{n} m\left(A_{h(n)}\right)$ $=0$ uniformly in $m \in \mathrm{M}$, which contradicts (1).

If $m: \mathcal{F} \rightarrow X$ is a finite additive measure with compact range, then the integration map $I_{m}: f \in \mathcal{B}(\mathcal{F}) \mapsto \int_{\Omega} f d m \in X$ is compact. In fact, in $\left[3\right.$, p. 263] it is proved that the sums of the form $\sum_{i=1}^{n} \alpha_{i} m\left(A_{i}\right), 0 \leq \alpha_{1} \leq$ $\cdots \leq \alpha_{n} \leq 1, A_{i} \cap A_{j}=\emptyset$ for $i \neq j$, belong to $\operatorname{co}(\operatorname{rg}(m))$. This yields the inclusion

$$
\left\{\int_{\Omega} f d m: f \in B_{\mathcal{B}(\mathcal{F})}\right\} \subset \overline{\mathrm{co}}(\operatorname{rg}(m))-\overline{\mathrm{co}}(\operatorname{rg}(m)) .
$$

Then the operator $I_{m}$ is compact.

Now we are ready to state our main result.

TheOREM 4.2. Let $\mathrm{M}$ be a subset of $\mathcal{M}_{\mathrm{c}}(\mathcal{F}, X)$. The following statements are equivalent:

(i) $\mathrm{M}$ is relatively compact.

(ii) $\mathrm{M}$ is equicompact and $\mathrm{M}(A)$ is relatively compact for all $A \in \mathcal{F}$.

Proof. (i) $\Rightarrow$ (ii). Put $\widehat{\mathrm{M}}=\left\{I_{m}: m \in \mathrm{M}\right\}$. As there is an isometry between $\mathcal{M}_{\mathrm{c}}(\mathcal{F}, X)$ and $\mathcal{K}(\mathcal{B}(\mathcal{F}), X)$ defined by $m \leftrightarrow I_{m}, \widehat{\mathrm{M}}$ is a relatively 
compact subset of $\mathcal{K}(\mathcal{B}(\mathcal{F}), X)$. By Theorem $\mathrm{A}, \widehat{\mathrm{M}}$ is equicompact and $\widehat{\mathrm{M}}(f)=\left\{\int_{\Omega} f d m: m \in \mathrm{M}\right\}$ is relatively compact, for all $f \in \mathcal{B}(\mathcal{F})$. So, in particular, (ii) holds.

(ii) $\Rightarrow$ (i). We consider the vector measure

$$
G: A \in \mathcal{F} \mapsto(m(A))_{m \in \mathrm{M}} \in \ell_{\infty}(\mathrm{M}, X),
$$

which has compact range since $\mathrm{M}$ is equicompact. Thus the integration map $I_{G}: \mathcal{B}(\mathcal{F}) \rightarrow \ell_{\infty}(\mathrm{M}, X)$ is compact and defined by $I_{G}(f)=\left(I_{m}(f)\right)_{m \in \mathrm{M}}$ for $f \in \mathcal{B}(\mathcal{F})$. From the compactness of $I_{G}$ it follows that $\widehat{\mathrm{M}}=\left\{I_{m}: m \in \mathrm{M}\right\}$ is equicompact. To prove that $\widehat{\mathrm{M}}$ is relatively compact in $\mathcal{K}(\mathcal{B}(\mathcal{F}), X)$, we only have to show that $\widehat{\mathrm{M}}(f)=\left\{\int_{\Omega} f d m: m \in \mathrm{M}\right\}$ is relatively compact for all $f \in \mathcal{B}(\mathcal{F})$. Given $f \in \mathcal{B}(\mathcal{F})$, choose a sequence $\left(\phi_{n}\right)_{n}$ of simple functions so that $f=\lim _{n \rightarrow \infty} \phi_{n}$ in $\mathcal{B}(\mathcal{F})$. Fix $\varepsilon>0$, and take $n \in \mathbb{N}$ such that $\left\|f-\phi_{n}\right\|<\varepsilon / s$, where $s=\sup \{\|m\|(\Omega): m \in \mathrm{M}\}$. For all $m \in \mathrm{M}$, we have

$$
\int_{\Omega} f d m=\int_{\Omega}\left(f-\phi_{n}\right) d m+\int_{\Omega} \phi_{n} d m \in \mathrm{M}\left(\phi_{n}\right)+\varepsilon B_{X},
$$

since $\left\|\int_{\Omega}\left(f-\phi_{n}\right) d m\right\| \leq \varepsilon$ for all $m \in \mathrm{M}$. It is obvious that $\mathrm{M}\left(\phi_{n}\right)$ is relatively compact, so we have proved that $\widehat{\mathrm{M}}(f)$ is relatively compact for all $f \in \mathcal{B}(\mathcal{F})$.

Corollary 4.3. Let $\left(m_{n}\right)$ be an equicompact sequence in $\mathcal{M}_{\mathrm{c}}(\mathcal{F}, X)$. If $\lim _{n \rightarrow \infty} m_{n}(A)$ exists for all $A \in \mathcal{F}$, then $\left(m_{n}\right)$ is convergent.

REMARK 4.4. A uniformly strongly additive set is not necessarily equicompact. For an example, take a noncompact and weakly compact subset $W$ of $L_{1}(\mu), \mu$ being Lebesgue measure on $[0,1]$. Denote by $\mathrm{M}(W)$ the set of indefinite integrals $\lambda_{f}=\int_{(.)} f d \mu$ with $f$ running over $W$. By [1, Th. VII.13], $\mathrm{M}(W)$ is uniformly countably additive, nevertheless, it is not equicompact in view of Theorem 4.2.

Examples of equicompact sets can be obtained in the following way: take a uniformly completely continuous set $\mathrm{N} \subset \mathcal{V}(Z, X), Z$ being an arbitrary Banach space, and a vector measure $m \in \mathcal{M}_{\mathrm{c}}(\mathcal{F}, X)$. It is easy to prove either directly or using Proposition 2.4 that the set $\mathrm{M}=\mathrm{N} \circ \mathrm{m}$ is equicompact.

TheOrem 4.5. Let $\mathrm{M}$ be a bounded subset of $\mathcal{M}_{\mathrm{c}}(\mathcal{F}, X)$. Then $\mathrm{M}$ is equicompact iff there exist a Banach space $Z$, a vector measure $m \in \mathcal{M}_{\mathrm{c}}(\mathcal{F}, X)$ and a uniformly completely continuous set $\mathrm{N} \subset \mathcal{V}(Z, X)$ so that $\mathrm{M}=\mathrm{N} \circ \mathrm{m}$.

Proof. We only have to prove the necessity. So, let $\mathrm{M} \subset \mathcal{M}_{\mathrm{c}}(\mathcal{F}, X)$ be bounded and equicompact. As in the proof of the above theorem, we can consider the vector measure

$$
G: A \in \mathcal{F} \mapsto(m(A))_{m \in \mathrm{M}} \in \ell_{\infty}(\mathrm{M}, X) .
$$


Again, the integration map $I_{G}: \mathcal{B}(\mathcal{F}) \rightarrow \ell_{\infty}(\mathrm{M}, X)$ is compact because $G$ has compact range. Moreover, $I_{G}(f)=\left(\int_{\Omega} f d m\right)_{m \in \mathrm{M}}$ for all $f \in \mathcal{B}(\mathcal{F})$. This proves that $\widehat{\mathrm{M}}=\left\{I_{m}: m \in \mathrm{M}\right\}$ is equicompact. According to Theorem 2.4, there exist a closed subspace $Z$ of $c_{0}$, an operator $Q \in \mathcal{K}(\mathcal{B}(\mathcal{F}), Z)$ and an equicompact set $\mathrm{N} \subset \mathcal{K}(Z, X)$ such that $\widehat{\mathrm{M}}=\mathrm{N} \circ Q$. If $m_{Q}$ denotes the representing measure of $Q$, we have $\mathrm{M}=\mathrm{N} \circ m_{Q}$. Finally, $m_{Q}$ is strongly additive because $Q$ is compact (see [3, Th. VI.1.1]).

\section{References}

[1] J. Diestel, Sequences and Series in Banach Spaces, Grad. Texts in Math. 92, Springer, 1984 .

[2] J. Diestel, H. Jarchow and A. Tonge, Absolutely Summing Operators, Cambridge Stud. Adv. Math. 43, Cambridge Univ. Press, Cambridge, 1995.

[3] J. Diestel and J. J. Uhl, Vector Measures, Math. Surveys Monogr. 15, Amer. Math. Soc., Providence, RI, 1977.

[4] J. Dieudonné, Foundations of Modern Analysis, Academic Press, New York, 1960.

[5] G. Emmanuele, A dual characterization of Banach spaces not containing $\ell_{1}$, Bull. Polish Acad. Sci. Math. 34 (1986), 155-160.

[6] H. Jarchow, Locally Convex Spaces, Teubner, Stuttgart, 1981.

[7] T. W. Palmer, Totally bounded sets of precompact operators, Proc. Amer. Math. Soc. 20 (1969), 101-106.

[8] H. P. Rosenthal, Pointwise compact subsets of the first Baire class, Amer. J. Math. 99 (1977), 362-378.

[9] E. Serrano, C. Piñeiro and J. M. Delgado, Equicompact sets of operators defined on Banach spaces, Proc. Amer. Math. Soc. 134 (2006), 689-695.

[10] K. Vala, On compact sets of compact operators, Ann. Acad. Sci. Fenn. Ser. A I 351 (1964), 47-55.

Departamento de Matemáticas

Facultad de Ciencias Experimentales

Campus Universitario del Carmen

Avda. de las Fuerzas Armadas s/n

E-21071 Huelva, Spain

E-mail: eserrano@uhu.es candido@uhu.es jmdelga@uhu.es 